livraisons

d'Histoire

de l'Architecture

\section{Livraisons de l'histoire de l'architecture}

$38 \mid 2019$

Varia III

\title{
Les écuries de chantilly : un palais à la gloire du cheval
}

The stables of Chantilly: a palace in the glory of the horse

Der Marstall von Chantilly. Ein Palast zu Ehren des Pferdes

\section{Christophe Levadoux}

\section{CpenEdition}

Journals

Édition électronique

URL : http://journals.openedition.org//ha/1472

DOl : $10.4000 /$ /ha. 1472

ISSN : 1960-5994

Éditeur

Association Livraisons d'histoire de l'architecture - LHA

Édition imprimée

Date de publication : 15 décembre 2019

Pagination : $39-49$

ISSN : $1627-4970$

Référence électronique

Christophe Levadoux, « Les écuries de chantilly : un palais à la gloire du cheval », Livraisons de l'histoire de l'architecture [En ligne], 38 | 2019, mis en ligne le 02 janvier 2021, consulté le 05 février 2021. URL: http://journals.openedition.org//ha/1472 ; DOI : https://doi.org/10.4000//ha.1472 
Par Christophe LeVadoux

\section{LES ÉCURIES DE CHANTILLY : UN PALAIS À LA GLOIRE DU CHEVAL}

Sébastien Mercier écrivait en 1781 : «Je n’ai encore rien trouvé de comparable à Chantilly, trente voyages dans ce lieu enchanté n'ayant pas encore épuisé mon admiration ; c'est le plus beau mariage qu'ont jamais fait l'Art et la Nature " ${ }^{1}$. Ces propos, quarante ans après la mort du duc de Bourbon (1692-1740), ne faisaient que confirmer les affirmations de Saint-Simon selon lequel : "Les sommes prodigieuses englouties à Chantilly par Henry-Jules ont été des bagatelles en comparaison des trésors que son petit-fils y a enterrés et merveilles qu'il y a faites ${ }^{2}$. En ce sens, la construction des écuries de Chantilly ${ }^{3}$ doit être replacée dans la perspective d'un pouvoir accru de M. le Duc face à Louis XV encore mineur, mais également dans une vaste vision urbanistique ayant pour objectif la transformation de l'ancien hameau en ville moderne.

\section{Un projet ancien enfin mis en ouvre}

Le projet de construire des écuries remontait à Henri-Jules de Bourbon-Condé (1643-1709), grand-père du duc de Bourbon, comme le montre une série de gravures d'Adam Pérelle (1640-1695) ${ }^{4}$. L'idée initiale établie par Jules HardouinMansart (1646-1708) prévoyait une cour en U à partir de l'Orangerie, dont il étendait les élévations à tous les nouveaux bâtiments. Lorsque le duc de Bourbon décida, très probablement en 1718 , de remplacer les anciennes écuries, médiocres installations logées dans l'ancienne ferme de Bucamp à Chantilly, il fit appel à Jean Aubert (1680-1741), dessinateur et collaborateur de Mansart. Aubert, qui s'était distingué

1. Sébastien Mercier, La Douceur de vivre, Neuchâtel, Samuel Fauche, 1781, n.p.

2. Louis de Rouvroy, duc de Saint-Simon, Mémoires, Paris, Hachette, 1873-1877, t. IV, p. 212.

3. Sur ces questions, on se réferera aux travaux de Pascal Liévaux, notamment: Pascal Liévaux, Les écuries des châteaux français, Paris, Éditions du patrimoine, 2005, 303 p. et Pascal Liévaux (dir.), Architecture équestre: Hauts-lieux dédiés au cheval en Europe, Actes Sud, 2010, 377 p.

4. Christophe Morin, Bâtiments d'utilité : accès et abords du château au XVIII siècle : le cas de l'Tlle-deFrance, thèse de doctorat, dactyl., Paris I, 2002, p. 192. 


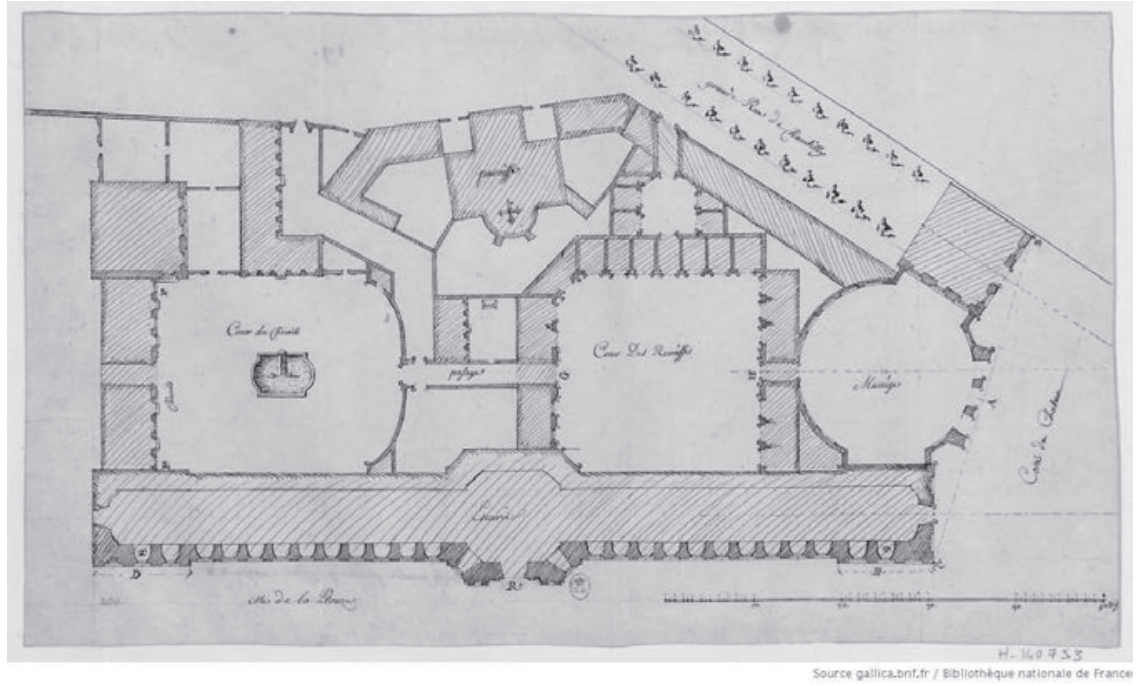

Ill. 1 : Attribué à Jean Aubert, Plan des écuries, remises, chenil et manège, 1719, H : 0,258 m ; L : 0,446 m, dessin, plume et encre de Chine, Paris, Bibl. nat. Fr., R. de C. 200, (C) Cl.Bibl.nat.Fr. Cet avant-projet proposant un ensemble de bâtiments placé entre la pelouse et la grand-rue est celui qui fut retenu dans la réalisation définitive.

à Saint-Maur, au Palais-Bourbon, ou à l'hôtel de Lassay à Paris 5 , lui livra plusieurs plans dès $1719^{6}$ (ill. 1).

Concrètement, les fouilles commencèrent à cette date, les bâtiments ne s'achevèrent qu'en 1735 , et le décor sculpté cinq ans plus tard. Il est à noter que toute la décoration fut exécutée entre 1734-1736, par et sous la direction de Rémy-François Bridault ${ }^{7}$. Totalement séparées du château, les écuries sont à Chantilly une puissance autonome, organisant leur propre paysage. En ne participant pas à la mise en scène

5. François Souchal, "Jean Aubert architecte des Bourbon-Condé", Revue de l'art, Paris, Ophrys, $\mathrm{n}^{\mathrm{o}}$ 6, 1969, p. 29-38.

6. BnF, Va 60 t. 12, R. de C. 200, Plan des écuries, remises, chenil et manège (avant-projet, 1719). Cet avant-projet proposant un ensemble de bâtiments placé entre la pelouse et la grand-rue est celui qui fut retenu dans la réalisation définitive ; Élévation de la façade du manège dudit château, et $d u$ pavillon à côté, 1719, Bibl.nat.Fr., Va 60 t. 12, R. de C. 201, sur ce premier projet, les trois baies du manège découvert sont ornées de bossages et surmontées d'une balustrade à trophées; dans le projet final, les baies comportent des colonnes engagées et la balustrade est ornée de gigantesques armoiries des Condé flanquées de deux licornes; Élévation du pavillon du milieu des écuries, 1719, Bibl.nat.Fr., Va 60 t. 12, R. de C. 202, cet avant-projet est très proche de la réalisation définitive ; il n'y manque que les œeils-de-bœuf pris dans la toiture et la Renommée en bronze qui la couronna ; Élévation de la porte de la cour des remises, 1719, Bibl.nat.Fr., Va 60 t. 12, R. de C. 203 ; Élévation de la moitié de la façade du chenil, du côté de la cour, 1719, Bibl.nat.Fr., Va 60 t. 12, R. de C. 204. Ces dessins sont également reproduits en double au musée Condé de Chantilly sous la cote $83 \mathrm{~L} 6 \mathrm{~A} \mathrm{r}^{\circ}$ et $83 \mathrm{~L} 6 \mathrm{~B}$.

7. Ibid. 
du château, Aubert innovait dans l'esprit comme dans la forme, même si quelques édifices avaient précédemment montré la voie, comme les écuries monumentales de Chaumont-La-Guiche en Bourgogne ou celles de Maisons ${ }^{8}$, construites toutes deux par François Mansart (1598-1666).

\section{Un ensemble aux apports et descendances plurielles}

Objet architectural isolé, le bâtiment d'Aubert intègre un système de références aux écuries les plus célèbres des XVII ${ }^{\mathrm{e}}$ et XVIII ${ }^{\mathrm{e}}$ siècles, savamment mises en scènes. En fait, l'héritage du vocabulaire architectural mis en œuvre par Hardouin-Mansart aux écuries de Versailles (1679-1685) ${ }^{9}$, à l'Orangerie de Sceaux (1685) ou à Chantilly $(1683)^{10}$, constitue le fondement du projet d'Aubert ${ }^{11}$.

L'utilisation de motifs entiers repris de Versailles, tels les chevaux cabrés à l'imposte du portail des écuries de Versailles et de Sceaux, furent autant d'emprunts indéniables. Cette réminiscence de l'architecture royale était significative de la volonté d'imiter le souverain, et d'inscrire les nouvelles constructions dans une perspective moderne. L'analogie qui peut être établie entre le programme des écuries de Chantilly et de Versailles souligne bien cette parenté. En effet, nous retrouvons chez Condé l'association de stalles avec un manège, un chenil, une école de pages ${ }^{12}$, et de nombreux logements comme à Versailles, où les deux bâtiments de part et d'autre de l'avenue de Paris avaient ces mêmes fonctions, bien que sous le simple rapport de la composition, et de la mise en scène urbaine du bâtiment, les deux projets aient été différents. Quant à l'idée du manège central elle vient de Richelieu ${ }^{13}$, où le pavillon central, seul bâtiment conservé de cet ensemble, formait un petit

8. Stockholm, National Museum, THC 472, Élévation et plan des écuries de Maison, v. 1660.

9. Dominique Massounie, L'Architecture des écuries royales, Paris, Nathan, 1998, p. 23-60 et plans du rez-de-chaussée de la Grande et de la Petite écurie de Versailles, BnF., Est Va 448 et 424 c. Voir du même auteur, "Les écuries jumelles de Versailles ", Alexandre Gady (dir.), Jules Hardouin-Mansart, Paris, Maison des Sciences de l'homme, 2010, 612 p.

10. L'Orangerie détruite de Chantilly était située face au Petit Château. La date de construction fut donnée par un dessin de l'agence Mansart, daté du $1^{\text {er }}$ janvier 1683 , BnF., est. Va 60 vol. (Fonds de Cotte).

11. Les différentes influences ont été reconnues par les auteurs suivants : Louis Hautecoeur, Histoire de l'architecture classique en France, Paris, Picard, 1950, t. III, p. 18, évoque la voûte de l'Orangerie de Versailles, et les écuries de Chaumont-Laguiche en Bourgogne; Jean-Marie Pérouse de Montclos, Histoire de l'architecture française, Paris, Éditions du Patrimoine, 1989, p. 331, affirme la filiation avec les écuries de Versailles; Jean-Pierre Babelon, Chantilly, Paris, Scala, 1999, p. 123, rapproche l'édifice de Versailles, comme Ernest de Ganay, Chantilly au XVIII e siècle, Paris-Bruxelles, Van Oest, 1925 , p. 33.

12. Charles Dugas, "À la cour des Condé Messieurs les pages ", Bulletin de la société d'histoire et d'archéologie de Senlis, Senlis, Imprimeries réunies, 1953, p. 12-13.

13. Jean Marot, Veüe perspective générale du château de Richelieu, ou de sa Basse-Cour, de son Anti-Cour, de sa cour et de ses aisles, Corps de Logis et Pasvillons, cité par Pascal Liévaux, Les écuries dans les châteaux français, Paris, Éditions du Patrimoine, 2006, fig. 69 p. 90 et p. 100. 
manège carré qui ne permettait sans doute que de faire travailler les chevaux à la longe.

La nouveauté de ce projet nous laisse penser qu'Aubert s'inspira plus particulièrement des écuries de Maisons, construites par François Mansart vers $1650^{14}$, pour René de Longueil (1596-1677), premier président de la Cour des Aides, qui reprenaient un principe entériné à Balleroy ${ }^{15}$, où la mise en scène des accès par la rue principale permettait directement une mise en scène du château. La monumentalité des écuries de Maisons correspondait aux vues du prince de Condé, même si leur implantation à angle droit avec le corps de logis inscrit le bâtiment dans un rapport de symétrie exclu à Chantilly. En fait, la distribution des jardins d'André Le Nôtre (1613-1700) organisés autour de la grande terrasse du château, empêchait tout bâtiment de venir détruire l'équilibre de la composition, bien que ce type d'écuries qui ne se voyaient pas depuis l'accès au château, ait été courant à Fontainebleau, Amboise, Villers-Coterêt ${ }^{16}$, ou à Oiron, depuis le XVI ${ }^{\mathrm{e}}$ siècle. Ajoutons que l'association d'une telle architecture avec le jardin, correspond à ce que fit Hardouin-Mansart à Sceaux, où il créa le même effet avec l'Orangerie.

La comparaison des deux bâtiments de Maisons et de Chantilly renforce l'originalité du parti adopté par Aubert. Mansart établit le plan monumental des écuries de Maisons à partir d'un axe de symétrie marqué par un imposant pavillon qui abritait en son centre un manège couvert expérimenté auparavant à Richelieu, et sur la partie postérieure formant une abside, un vaste abreuvoir richement décoré à la manière des grottes italiennes. Aubert fonda également la grande nef des écuries de Chantilly sur la symétrie du dôme central (ill. 2) qui, à l'imitation de Mansart, accueillait une fontaine dont le décor luxueux et naturaliste était tout à fait dans le goût du temps ${ }^{17}$.

L'analyse des plans des ensembles de Maisons et de Chantilly montre une évolution de l'organisation des stalles, plus rationnelle chez Aubert ${ }^{18}$. S’il appliqua la symétrie à chacune des parties de l'édifice (cours et façades en particulier), l'architecte ne respecta pas une régularité absolue du plan d'ensemble. Il intègre au complexe des nouvelles écuries, l'ancienne église paroissiale de Jules Hardouin-Mansart.

14. George Poisson, "Recherche sur les écuries de Maisons", Bulletin de la Société de l'Histoire de l'Art français, Paris, Société de l'Histoire de l'Art français, 1980, p. 81-92; Claude Mignot, "Le château de Maisons 1641/1650/1658 et 1660 ", Paris, éditions du Patrimoine, 2013, 48 p. ; Jean-Pierre Babelon et Claude Mignot, François Mansart, Paris, Gallimard, 1998, p. 175-179.

15. Louis Bourdon, Vue et perspective du Château de Basleroy, 1715 ; cité par Pascal Liévaux, Les écuries dans les châteaux français, op. cit., p. 97, fg. 78 .

16. Androuet Du Cerceau, Les plus excellents Bastiments de France, Paris, Sand \& Conti, 1988, p. 179, $187,199$.

17. Jacques Mérigot, Promenade ou itinéraire des jardins de Chantilly, Paris, Desenne, 1791, p. 55.

18. Christophe Morin, Bâtiments d'utilité : accès et abords du château au XVIII siècle : le cas de l'Île-deFrance, op. cit., p. 195. Le plan des écuries de Maisons peut être rapproché en particulier de celui des thermes dans lesquels se manifesta la même volonté de composer à partir de pièces aux formes régulières. Du même auteur, voir également à ce sujet, Au service du château, L'architecture des communs au XVIII siècle, Paris, éditions de la Sorbonne, 2008, p. 247-273. 


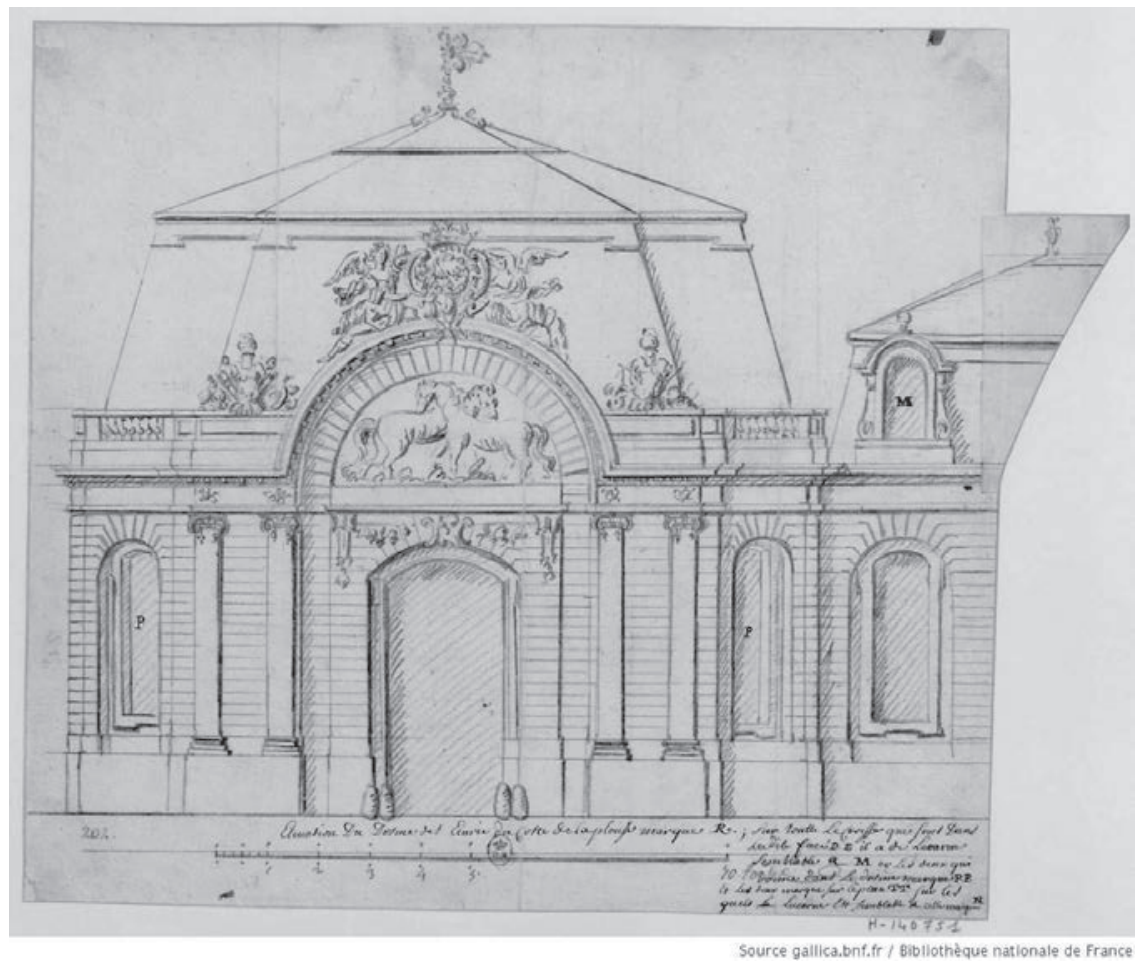

Ill. 2 : Jean Aubert, Elévation du dosme des Ecuries..., mine de plomb, 1719, dimensions non indiquées, Paris, Bibl. nat. Fr., Va 60 t 12, (c) Cl. Bibl. nat. Fr.

Les parties visibles offrent ainsi une régularité qui masque l'asymétrie des bâtiments du côté du village.

Tant à Maisons qu'à Chantilly ${ }^{19}$, les architectes distribuèrent deux cours derrière les façades monumentales (ill. 3), où sont disposées les remises (ill. 4). Il est bien un point commun entre les écuries de Versailles et de Chantilly, c'est l'ensemble des contraintes induites par l'irrégularité des parcelles sur lesquelles les bâtiments ont été construits. Les deux architectes utilisent la fameuse formule de la rotule distributive afin de ménager les communications entre les différentes galeries des écuries de Versailles, et rattraper ainsi l'angle disgracieux formé par le château et les écuries à Chantilly. Les élévations à Chantilly forment, quant à elles, un paravent monumental à la ville, qu'elles isolent visuellement du château. Chacune des trois façades principales des écuries du prince de Condé reçut un décor différent, en rapport avec les fonctions du complexe. Leur orientation manifestait la volonté d'insérer la

19. Un plan anonyme des écuries de Maisons conservé à Stockholm (CC 113), montre sept remises, implantées à la périphérie des cours ; cité par Christophe Morin, Bâtiments d'utilité : accès et abords du château au XVIII ${ }^{e}$ siècle: le cas de l'île-de-France, op. cit., p. 195, note $\mathrm{n}^{\circ} 844$. 


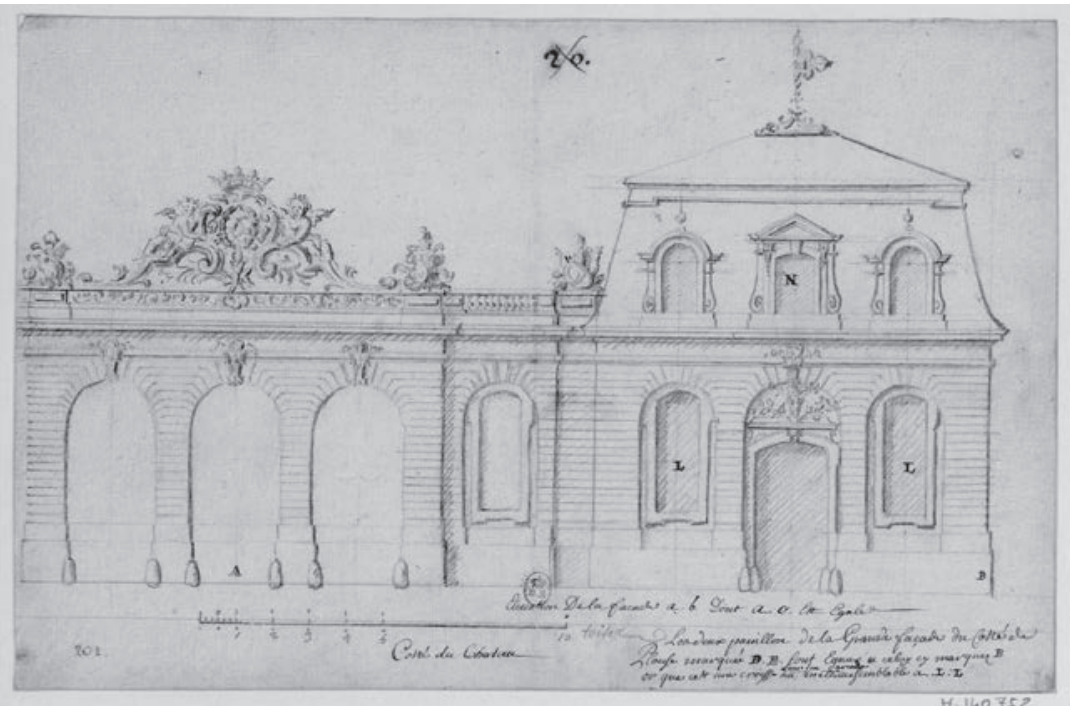

Source gatlica.bnt,fr / Eblichteque nationsle de France

Ill. 3 : Jean Aubert, Elévation de la façade du manège du château, et du pavillon à côté, 1719, L : 0,207m ; 1: 0,322 m, dessin, pierre noire, Paris, Bibl. nat. Fr., R. de C. 201, (C) Cl. Bibl. nat. Fr.

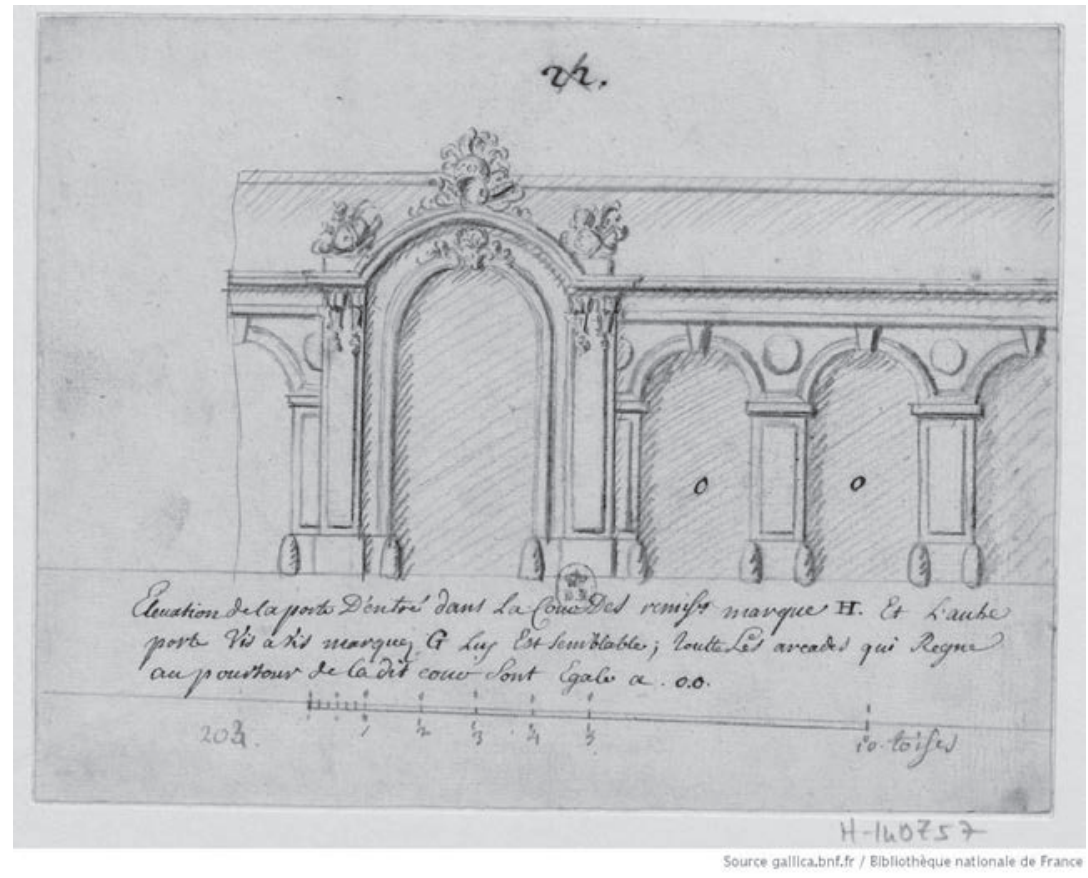

Ill.4 : Jean Aubert, Elévation de la porte de la cour des remises, 1719, L: 0,16m ; 1: 0,208 m, dessin, pierre noire, Paris, Bibl. nat. Fr., R. de C. 203, (C) Cl. Bibl. nat. Fr. 
nouvelle construction dans le tissu urbain existant. Pour se faire, elles s'inscrivirent sur un axe parallèle au canal de Saint-Jean, qui apparaissait sur les plans de Chantilly dès 1673 , et reliait l'hôpital à l'église paroissiale et à l'ancienne ferme de Bucamp. Si le décor des façades renvoie bien au vocabulaire utilisé par Jules Hardouin-Mansart, l'élève surpassa l'œuvre du maître par les proportions du bâtiment, comparables à celles de Maisons. De la même manière, les proportions monumentales et harmonieuses des écuries de Chantilly vinrent suppléer la silhouette irrégulière du vieux château en contrebas, et fonctionnèrent comme le point de mire du domaine, éclipsant du même coup le corps de logis. Une gravure de Pérelle montre l'effet produit par ce rapport des masses original entre les écuries et le château de Maisons, même si la déformation de la gravure est à prendre en compte ${ }^{20}$. En effet, la façade latérale sur les fossés secs du château offrait comme à Chantilly une vue biaise depuis le corps de logis. La présence de colonnes et pilastres sur les façades cantiliennes et de Maisons représente un autre point commun. Défiant la " convenance » classique de Jacques-François Blondel (1705-1774) ${ }^{21}$, appuyés en cela par l'ordre ionique des avant-corps et du manège, ils étaient absents de Versailles sur les logis comme sur les communs. En effet, les bâtiments estimés par le maître n'avaient pas besoin d'un tel décorum, et devaient se suffire à eux-mêmes ${ }^{22}$. Les critiques à l'égard des écuries, essentiellement d'ordre esthétique, impliquent-elles également un ou des défauts de construction ${ }^{23}$ ? La réponse ne peut-être que positive, Blondel en reprochant à Aubert de leur avoir donné trop de hauteur, sous-entendait également que le froid y régnait souvent. De même, en optant pour le passage unique entre les deux rangs, il tourna les chevaux face aux ouvertures dont le jour endommage les yeux ${ }^{24}$.

Si le projet d'Aubert fut respecté dans ses grandes lignes, l'état actuel reflète quelques changements de parti par rapport aux projets initiaux. Tout d'abord en ce qui concerne le pavillon central : selon Piganiol (1669-1753), l'entablement de la façade présentait, de part et d'autre de la porte principale, le chiffre du prince de Condé. Nous pouvons toutefois nous demander si cet auteur montrait la réalité, ou s'il se contentait, comme il le fit à plusieurs reprises, de décrire un projet. En effet, sur le dessin préparatoire figure bien le chiffre LH, alors que ces motifs n'existent ni sur l'édifice actuel, ni sur les dessins de l'Album du comte du Nord (1782). D'autres différences jalonnent encore l'ouvrage. Bien que le larmier soit porté par deux consoles et un cartouche, celui-ci ne correspond pas exactement au modèle exécuté. Par ailleurs, il n'y a que deux chevaux sur le tympan, au lieu des trois actuels.

20. A contrario, nous pouvons estimer que l'exagération des proportions par le dessinateur servit à montrer l'effet voulu par l'architecte. À ce titre, voir Jean-Pierre Babelon et Claude Mignot, François Mansart, op. cit., p. 178.

21. Werner Szambien, Symétrie. Goût. Caractère. Théorie et terminologie de l'architecture à l'âge classique. 1500-1800, Paris, Picard, 1986, p. 169-173.

22. Jacques-François Blondel, Cours d'architecture, Paris, Desaint, 1771, t. I, p. 391.

23. Dominique Massounie, "Le logement des chevaux aux XVII ${ }^{\mathrm{e}}$ et XVIII ${ }^{\mathrm{e}}$ siècles : Paris, Maisons, Versailles et les recommandations des architectes au XVIII ${ }^{\mathrm{e}}$ siècle ", Livraisons d'Histoire de l'Architecture, $\mathrm{n}^{\mathrm{o}} 6,2^{\mathrm{e}}$ semestre 2003, p. 69-86.

24. Ibid., p. 81. 


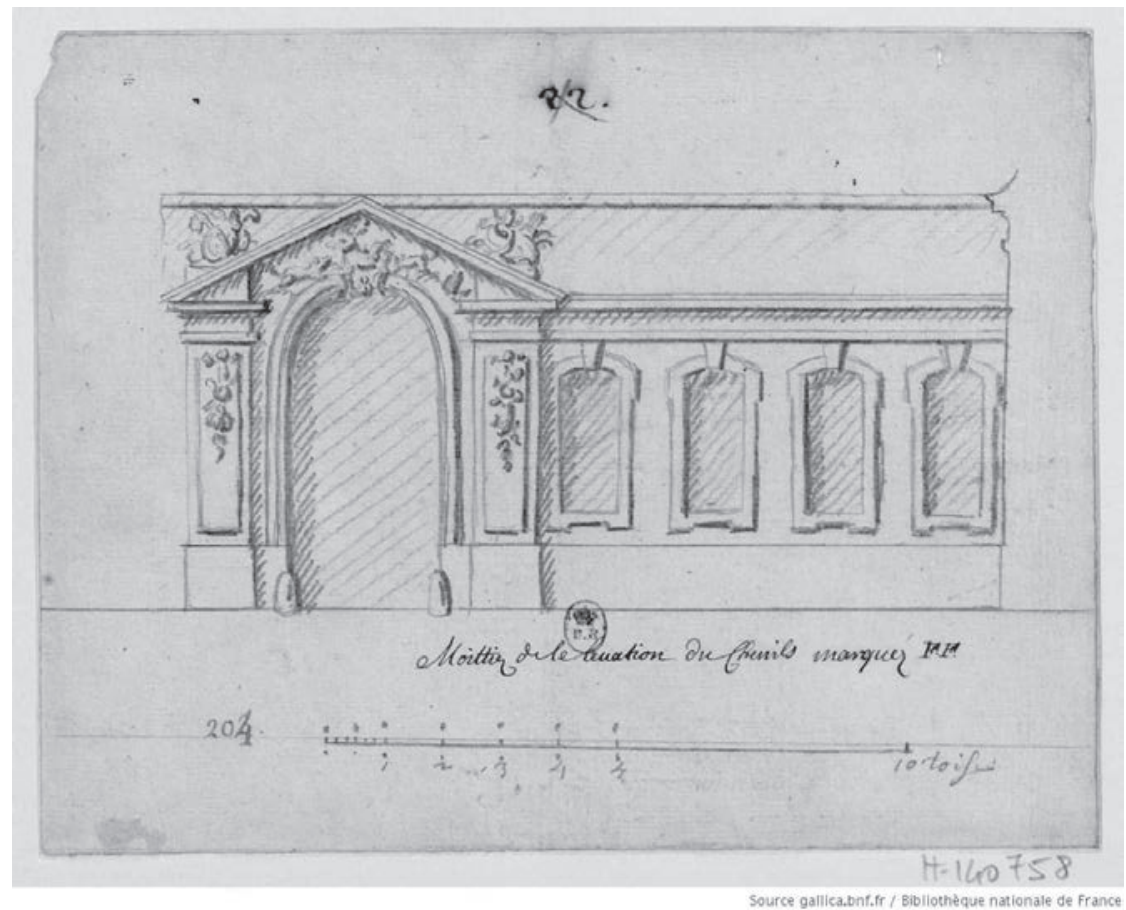

Ill. 5 : Jean Aubert, Elévation de la moitié de la façade du chenil, du côté de la cour, 1719, L: 0,162 m ; 1: 0,206 m, dessin, pierre noire, Paris, Bibl. nat. Fr, R. de C. 204, (C) Cl. Bibl. nat. Fr.

Mais surtout, sur le projet, pourtant très proche du parti définitif, il manque les œils-de-bœuf pris dans la toiture, et la figure de la Renommée qui la couronne. De surcroît, Aubert avait d'abord imaginé un manège sans colonnes, simplement orné de refends. L'entablement était pourvu d'un écu retenu par deux anges, flanqué de deux trophées à l'emplacement actuel des chevaux. Quant au pavillon inachevé, Piganiol le décrit dans son état achevé, lequel figure également dans un état contemporain, conservé dans les collections du musée Condé. Le même état inachevé se retrouve par ailleurs au niveau de l'entrée de la cour des chenils (ill. 5) et de la cour des remises. En effet, le revers de leur façade fut laissé à l'état d'épannelage, alors que ces morceaux en attente étaient figurés sculptés.

L'exemple des écuries de Chantilly connut dès la seconde moitié du XVIII ${ }^{\mathrm{e}}$ siècle une diffusion importante. En France, les écuries de La Roche-Guyon, Bizy, Montgeoffroy, de Craon, ou dans une certaine mesure, celles des Conti à l'Isle-Adam, présentaient une analogie certaine. Le cas de La Roche-Guyon (1745-1747) nous en fournit un bon exemple. Commencé en 1725 sur ordre de François de La Rochefoucauld $(1633-1728)^{25}$, le chantier se prolongea jusqu'en 1739. Ce processus engagé par le

25. Arch. dép. du Val-d'Oise, $10 \mathrm{~J} 456$; cité par Christophe Morin, Bâtiments d'utilité : accès et abords du château au XVIII e siècle : le cas de l'T̂le-de-France, op. cit., p. 209, note 912. 
duc, poursuivi par sa veuve après 1728, et achevé par son fils Armand (1690-1762) avec la cour des écuries, peut être mis en parallèle avec la démarche de Condé à Chantilly. Les deux domaines symbolisent la noblesse de familles illustres. Les écuries de Bizy (1769) occupent une place à part dans ce que nous pourrions appeler les successeurs de Chantilly $^{26}$. En effet, leur situation particulière ne répond pas en totalité au modèle versaillais, et intègre plus explicitement la mise en scène présente chez le prince de Condé auquel Pierre Contant d'Ivry (1698-1777) emprunta l'idée d'une archivolte grandiose ${ }^{27}$, pour marquer le point de symétrie de l'ensemble, souligné par ailleurs par un dôme à pans, sur la partie arrière de la cour ${ }^{28}$. La cour des écuries est fermée par un bâtiment monumental transformé en logis, dans l'attente de la reconstruction de l'ancien château vétuste, flanqué de deux rotondes, et marqué en son centre par un portail monumental en forme d'arc de triomphe, encadré de pilastres doriques colossaux, souligné par une toiture indépendante en pavillon ${ }^{29}$. Néanmoins, la façade monumentale qui fermait la basse-cour du côté de la cour d'honneur, rythmée par un registre d'arcades en plein cintre, soulignées par une ligne d'imposte, et timbrées de mascaron, rappelle clairement Versailles. Comme à Chantilly, l'exemple de Maisons reste présent avec l'utilisation de toitures indépendantes pour chaque partie de ce bâtiment. Par ailleurs, l'architecte créa des modèles de hauts-reliefs inspirés par l'iconographie des petites écuries de Versailles où, dans l'archivolte, un cocher mène une tige de chevaux cabrés. La planche XLVII du recueil de Contant d'Ivry présente un sujet similaire, librement inspiré par ce modèle. Dans le même ordre d'esprit, Gizeux déploie aussi une ample composition architecturale qui marque le paysage. L'aile des écuries et celle des remises en retour contribuent au premier chef à la mise en scène du pouvoir et du mode de vie aristocratiques, au même titre que la vieille tour médiévale conservée à l'entrée ${ }^{30}$; alors qu'à Montgeoffroy, les écuries sont accessibles par une porte ménagée à l'intérieur du passage charretier qui les sépare également du château. En ce qui concerne Craon, d'une architecture très simple mais parfaitement symétrique, les écuries ne sont comparables à celles de Chantilly, construites un demi-siècle auparavant, que par leur parti distributif axé sur un manège de plan centré d'environ treize mètres cinquante sur treize, qui s'élève sur toute la hauteur de l'édifice, laissant apparaître la charpente du toit ${ }^{31}$. Soulignons que la façade principale des écuries fut, à ce

26. Gustave Taraval, "Projet de la principale entrée des écuries du château de Bizy " et "Autre projet de l'entrée d'un manège pour les basses-cours du château de Bizy ", in Euvres d'architecture de Pierre Contant d'Ivry, 1769, pl. 50 et 25 ; cité par Pascal Liévaux, Les écuries dans les châteaux français, op. cit., p. 171, fig. 166. Et "Château de Bizy. Plan général du château ", 1845 ; ibid., p. 179 , fg. 174.

27. Pierre Contant d'Ivry, Les Euvres d'architecture, Paris, Huquier, 1769, p. 12.

28. Louis Hautecœur, Histoire de L'Architecture classique en France, op. cit., t. III, p. 229.

29. Ce bâtiment a disparu, remplacé par l'actuel château construit sous Louis-Philippe (1773-1850). L'élévation du corps de bâtiments qui fermait la cour des écuries ne nous est connue que par un dessin conservé aux Archives départementales de l'Eure, et publié par Jean-Louis Baritou et Dominique Foussart, Chevotet, Contant, Chaussard, Lyon, La Manufacture, 1987, p. 117.

30. Pascal Liévaux, Les écuries dans les châteaux français, op. cit., p. 192.

31. Ibid., p. 169. 
titre, traitée à l'économie, la disposition régulière des ouvertures étant soulignée par les encadrements de brique, et l'axe marqué par la grande porte qui donne accès au manège. Construites sur les plans de Jean-Baptiste André en $1777^{32}$, les écuries du château de l'Isle-Adam reprirent l'évolution d'un ensemble monumental au sein d'un tissu urbain. La citation des héritages cantilien et versaillais ne se cantonnait pas à ces seules données. En effet, selon le modèle versaillais ${ }^{33}$, les bâtiments se répartissaient autour d'une patte-d'oie, dont les avenues s'étendaient jusqu'à l'horizon. Dans le but d'agrandir la perspective du château, le prince de Conti fit percer dans l'axe une grande avenue de marronniers, et forma la troisième aile de la patte-d'oie. Les bâtiments, séparés au milieu par l'allée de marronniers, présentaient la forme de deux ailes concaves ${ }^{34}$, percées d'arcades pour les remises, flanquées de pavillons, qui encadraient une vaste cour de forme trapézoïdale. À l'arrière, deux bâtiments longitudinaux abritaient les écuries proprement dites, isolées du château. Face au château, l'élévation des ailes montrait un registre d'arcades en plein cintre, surmonté d'un attique, et du fameux décor à lignes de refends, présent aux orangeries de Sceaux et de Versailles. En outre, les pavillons arrière, dont la couverture en comble brisé rappelait une nouvelle fois Versailles, présentaient un beau décor sculpté par Moitte dans les impostes des arcades. Les chevaux cabrés, projetés dans les archivoltes des pavillons des écuries de l'Isle-Adam ${ }^{35}$, s'ils sont selon Élyne Olivier ${ }^{36}$, un emprunt à Chantilly, renvoient directement au modèle d'Hardouin-Mansart, sculpté par François Girardon (1628-1715) à Versailles. Mais il est certain que des motifs comme le pavillon à dôme carré ou les lignes de refends répandues sur les façades, renvoyaient directement aux modèles de Mansart et Jacques Gabriel (1667-1742) ${ }^{37}$. En outre, en ce qui concerne le logement des chevaux, nous retrouvons dans le plan d'André la majesté des grandes écuries princières. C'est en tout cas sur le modèle cantilien, qu'André conçut l'élévation intérieure de ses écuries, où nous retrouvons la même ponctuation par les pénétrations qui dégagent les baies du bâtiment. Néanmoins, la grande originalité de la composition intérieure des écuries réside ici dans le rythme donné à l'élévation par des colonnes toscanes engagées au tiers, audace que ni Mansart ni Aubert n'avaient pas osée.

32. Michel Gallet, Les Architectes parisiens du XVIII siècle, Paris, Mengès, 1995, p. 23 et Edmond Olivier-Valengin, "Le château des princes de Bourbon-Conti à l'Isle-Adam ", Trésors des princes de Bourbon Conti, catalogue d'exposition sous la direction de Frédéric Chappey, Paris/L'Isle-Adam, Somogy/Musée d'art et d'histoire Louis Senlecq, 2000, p. 122. Jean-Baptiste André, "Plan général du château et du village de l'Isle-Adam ", Chronologie (...), (planche non paginée) ; "Plan général et élévation des écuries du château de l'Isle-Adam ", 1809, Chronologie (...), planche première (non paginée) ; cité par Christophe Morin, «Les écuries de l'Isle-Adam : chant du cygne des princes de Conti ", Livraisons d'Histoire de l'Architecture, $\mathrm{n}^{\circ}$ 6, $2^{\mathrm{e}}$ semestre 2003, p. 91 et 93, Ill. 2 et 3.

33. Jean-Baptiste André, "Plan général des élévations des écuries du château de l'Isle-Adam », 1809, Chronologie (...), planche première (non paginée) ; ibid., p. 91, Ill. 2.

34. Jean-Baptiste André, Élévations des pavillons des écuries de l'Isle-Adam, 1778, Arch. nat., NII Seineet-Oise $196^{16}$; ibid., p. 93, Ill. 3 .

35. Ibid.

36. Edmond Olivier-Valengin, "Le château des princes de Bourbon-Conti à l'Isle-Adam ", op. cit., p. 122.

37. Christophe Morin, "Les écuries de l'Isle-Adam : chant du cygne des princes de Conti ", op. cit., p. 92 . 
Cette approche des écuries du duc de Bourbon, montre la place occupée par le cheval dans les préoccupations de l'aristocratie. Elle en souligne l'originalité et l'importance. Le cheval se trouve au cœur d'un ensemble, qui avec la mise en place d'un nouvel urbanisme, regroupe et fait vivre plusieurs centaines de personnes. L'importance des sommes consacrées à cette consommation, la diversité des interventions que cela sous-tendait, témoignent du soin porté à l'entretien et à l'organisation, ainsi que des mouvements perpétuels qui animèrent la maison princière ${ }^{38}$.

Christophe LEVADOUX Docteur en histoire de l'art moderne Enseignant, chargé de cours en classes préparatoires

38. Voir Christophe Levadoux, "L'organisation socio-économique des écuries de chantilly durant la première moitié du XVIII ${ }^{\mathrm{e}}$ siècle ", In Situ, rubrique Varia, n 12 , février 2010. 
